\title{
A Cutaneous Lesion Associated With Primary Polymyositis
}

Francisco Indart, MD

Department of Internal Medicine

Agustin España, MD

Department of Dermatology

Miguel A. Idoate, MD

Department of Pathology

Ignacio Lucas, MD

Department of Internal Medicine

Emilio Quintanilla, MD

Department of Dermatology

University Clinic of Navarra. School of Medicine. 31080 Pamplona, Spain

Polymyositis is a nonsuppurating inflammatory disease of the striated muscle characterized clinically by the presence of proximal muscular weakness that is sometimes associated with pain. ${ }^{1}$ This disease falls within a broader category known as idiopathic inflammatory myopathy, which includes the forms of myositis that are secondary to connective tissue disease and neoplasms and myositis with inclusion bodies. ${ }^{2}$ Polymyositis is differentiated from dermatomyositis by the absence of the characteristic cutaneous lesions. ${ }^{1}$

Stahl et $\mathrm{al}^{3}$ reported the presence of hyperkeratosis of the fleshy pad and the lateral aspect of the fingers to be a marker for idiopathic inflammatory myositis. We describe a woman with primary polymyositis associated with hyperkeratosis of the fingers of both hands.

\section{REPORT OF A CASE}

A 55-year-old housewife presented in August 1991 with arthromyalgia, predominantly in the-upper limbs, affecting the shoulders, wrists, and metacarpophalangeal and proximal interphalangeal joints of both hands. She also complained of having a sensation of loss of strength. The patient also reported the parallel development of fissures and thickening of the distal portion of the fingers of both hands.

After treatment with nonsteroidal antiinflammatory drugs, the polyarthralgia improved, but the fingertip lesions remained unchanged. The patient later noted generalized muscular weakness with stiffness, pain, and heaviness of the lower extremities that limited her ability to perform household tasks, climb stairs, and sit in a chair. During this second phase, edema of the feet and legs appeared. There were no other cutaneous 
manifestations. Raynaud's phenomenon or other associated systemic syndromes were absent. The patient had not previously undergone any pharmacologic treatment.

Physical examination revealed an overall reduction in strength in all limbs, with slow gait and great difficulty in walking on her heels. The osteotendinous reflexes were preserved. Pain was also experienced in the anterior aspect of the thighs on flexion of the knees, and tenderness was noted on pressure and palpation of the muscular masses in both legs. The hands felt hot, and no signs of arthritis were present. There was diffuse tumefaction of the fingers. Diffuse edema was observed on the feet and legs. Also noted was the presence of fissured yellow hyperkeratotic lesions on the fleshy pads and distal lateral surfaces of the fingers of both hands, chiefly in the index fingers (Figure 1). No abnormality of the nails was observed. We found no other skin lesions worthy of mention.

The most relevant analytic data were as follows: leukocytes, $7.6 \times 10^{9} / \mathrm{L}$, with normal cell differential; serum oxaloacetic transaminase, $361 \mathrm{U} / \mathrm{L}$ (normal, $<21 \mathrm{U} / \mathrm{L}$ ); serum pyruvic transaminase, $430 \mathrm{U} / \mathrm{L}$ (normal, $<22 \mathrm{Ul} / \mathrm{L}$ ); aldolase, $2.3 \mathrm{U} / \mathrm{L}$ (normal, 0.1 to $3.1 \mathrm{UVL}$ ); creatine phosphokinase, $4780 \mathrm{U} / \mathrm{L}$ (normal, <110 U/L); and lactic dehydrogenase, $2108 \mathrm{U} / \mathrm{L}$ (normal, 160 to $320 \mathrm{U} / \mathrm{L}$ ). The following tests proved normal or negative: SMAC-12 (12-channnel sequential multiple analyzer with computer), urine analysis, antinuclear antibodies, antibodies to extractable nuclear antigens, antibodies to Jo-1 antigens (histidyl-tRNA synthetase), and thyroid hormones. The following serology tests proved negative: Trichinella spiralis, Toxoplasma, hepatitis virus B and $\mathrm{C}$, influenza virus $\mathrm{A}$ and $\mathrm{B}$, parainfluenza virus 1,2 , and 3, and coxsackievirus $\mathrm{A}, \mathrm{B} 1$, $\mathrm{B} 2, \mathrm{~B} 3, \mathrm{~B} 4, \mathrm{~B} 5$, and $\mathrm{B} 6$.

A chest roentgenogram and electrocardiogram were normal. The electromyogram showed primary myopathic anomalies. A muscle biopsy specimen revealed muscular atrophy with a dense inflammatory lymphoplasmocytic infiltrate in the perimysium. The biopsy specimen of the skin lesions on her fingers revealed compact irregular orthokeratotic hyperkeratosis, alternating with areas of basket-weave keratin, intact granulous layer, irregular acanthosis, isolated dyskeratotic keratinocytes, and lymphocytic epidermotropism. A dense strip of lymphocytic infiltrate was seen in the superficial dermis (Figure 2). Prednisone was given at an initial dose of $1.5 \mathrm{mg} / \mathrm{kg}$ per day, and the patient reported a spectacular recovery with regard to the feeling of weakness and muscular pain. The fingertip lesions disappeared within a few days. The dose of corticosteroids was then gradually tapered to a maintenance dose of $10 \mathrm{mg} / \mathrm{d} 4$ months after treatment commenced. The hyperkeratotic lesions on the fingertips did not reappear within this time.

\section{COMMENT}

Stahl et $\mathrm{al}^{3}$ described eight patients with diseases of the connective tissue (four with mixed-connective tissue disease, three with dermatomyositis, and one with systemic lupus erythematosus) presenting inflammatory myositis in association with hyperkeratotic lesions of the fleshy pads and the lateral aspect of the fingers of both hands. These authors suggest that the findings of these skin lesions may be useful in the detection and diagnosis of idiopathic inflammatory myositis. More recently, Callen ${ }^{1}$ has 
stated that, up until 1987, no new cases of idiopathic inflammatory myositis associated with such skin lesions had been reported.

In our patient, it is striking that the skin lesions and muscular symptoms were concomitant. This phenomenon suggests a possible relationship between these two processes. In the patients described by Stahl et al, ${ }^{3}$ the finger lesions did not disappear when topic or systemic corticosteroids were given. Nonetheless, these authors do not report the actual dose given. In our case, both processes cleared when high doses of corticosteroids were administered $(1.5 \mathrm{mg} / \mathrm{kg}$ per day).

We cannot be certain of the meaning of the histopathologic finding from the skin lesions in our patient. It is difficult to reach any conclusions in view of the absence of such studies in the cases reported previously. ${ }^{3}$

As Stahl et $\mathrm{al}^{3}$ maintain, the presence of these highly characteristic skin lesions on the fingertips may be a marker for connective tissue disease associated with inflammatory myositis. The fact that no further cases have been reported in the literature to date may, perhaps, result from a lack of knowledge of this association; skin lesions of this type may well have been confused with other processes, such as reactive frictional hyperkeratosis, contact eczema, or dyshydrotic eczema.

\section{REFERENCES}

1) Callen JP. Dermatomyosins. Dis Mon. 1987; 33: 237-305.

2) Plotz PH, Dalakas M, Leff RL, et al. Current concepts in the idiopathic inflammatory myopathies: polymyositis, dermatomyositis and related disorders. Ann Intern Med. 1989; 111: 143-157.

3) Stahl NL, Klippel JH, Decker JL. A cutaneous lesion associated with myositis. Ann Intern Med. 1979; 91: 577-579. 


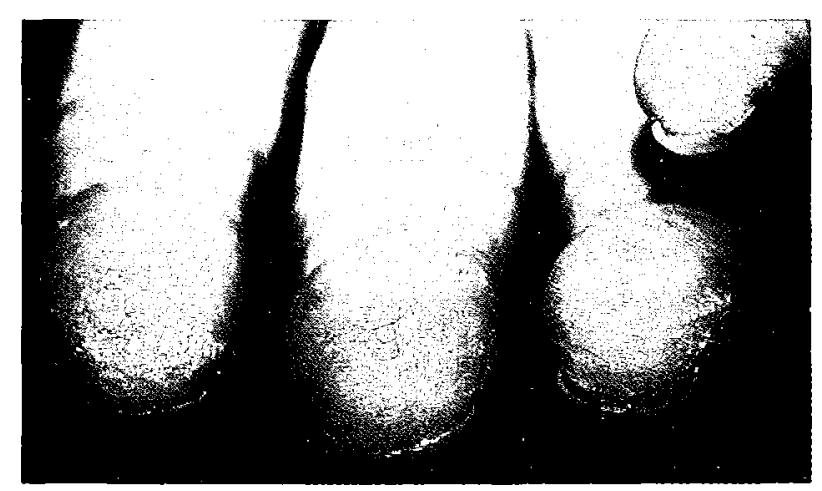

Figure 1. Fissured, yellowish, hyperkeratotic lesions located on the fingertips and distal lateral portions of the fingers.

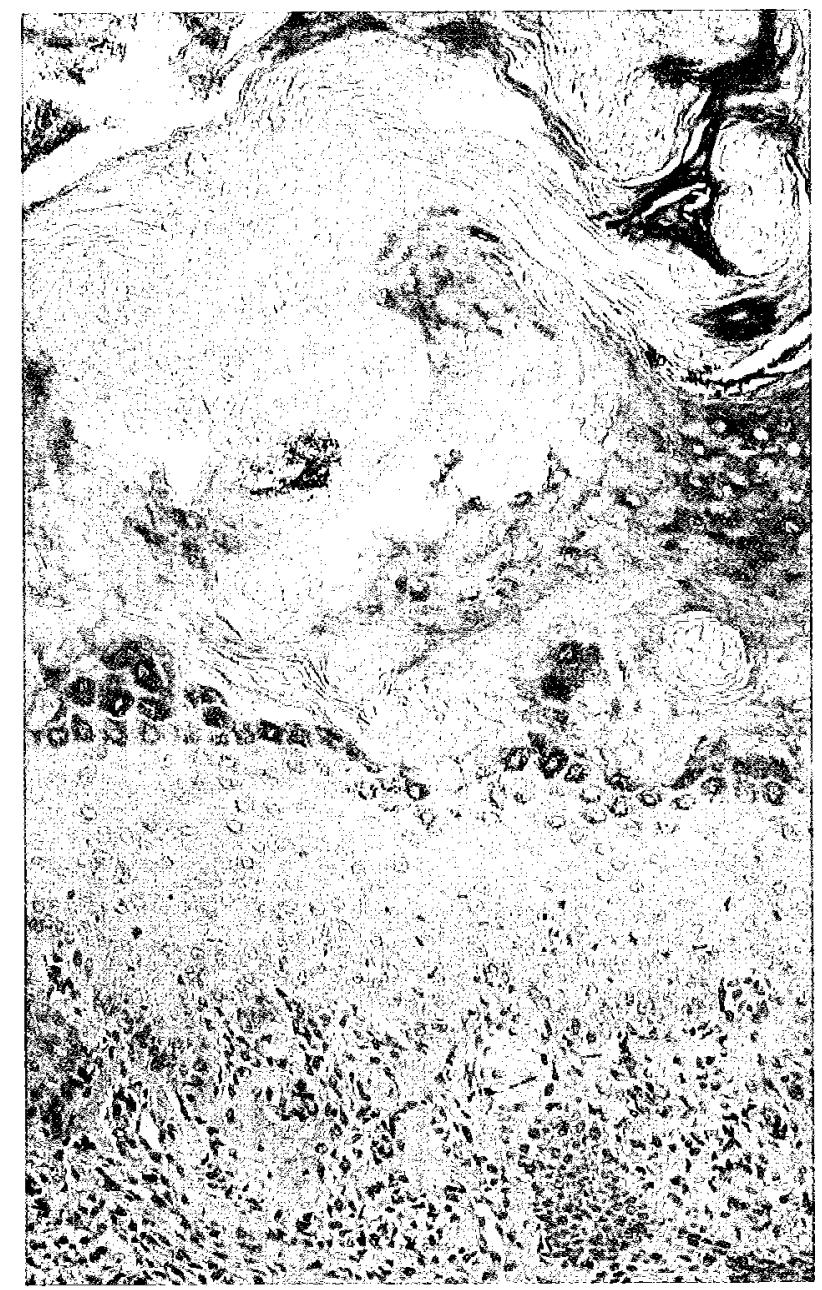

Figure 2. Orthokeratotic hyperkeratosis, irregular acanthosis, isolated dyskeratotic keratinocytes, and strip-shaped lymphoplasmocytic infiltrate with areas of epidermotropism all seen in the superficial dermis (hematoxylin-eosin $x 200$ ). 